\title{
Integrable Models of Interaction of Matter with Radiation ${ }^{\star}$
}

\author{
Vladimir I. INOZEMTSE $V^{\dagger}$ and Natalia G. INOZEMTSEVA $\ddagger$ \\ $\dagger$ Laboratory of Theoretical Physics, JINR, Dubna, Russia \\ E-mail: inozv@theor.jinr.ru \\ ¥ Moscow Technical University, Dubna Branch, Russia
}

Received July 18, 2006, in final form September 19, 2006; Published online October 13, 2006

Original article is available at http://www.emis.de/journals/SIGMA/2006/Paper069/

\begin{abstract}
The simplified models of interaction of charged matter with resonance modes of radiation generalizing the well-known Jaynes-Cummings and Dicke models are considered. It is found that these new models are integrable for arbitrary numbers of dipole sources and resonance modes of the radiation field. The problem of explicit diagonalisation of corresponding Hamiltonians is discussed.
\end{abstract}

Key words: integrability; radiation; Gaudin models

2000 Mathematics Subject Classification: 81R05; 81S99

\section{Introduction}

In the last four decades, much attention has been paid to the problem of describing processes of interaction of charged matter with electromagnetic radiation at resonance [1, 2, 3, 4]. This problem, being overcomplicated from the mathematical viewpoint in the general case, admits some very attractive simplifications which allow one to construct some rather simple models having even exact solutions. These models can be used for quantum statistical description of real interaction processes.

The starting point for constructing such models is the quantum Hamiltonian [3]

$$
H=\frac{1}{2 m}\left(\vec{p}-\frac{e}{c} \vec{A}\right)^{2}+u(x)+H_{F}, \quad H_{F}=\sum_{k} \omega_{k}\left(b_{k}^{+} b_{k}+\frac{1}{2}\right),
$$

where $\vec{p}$ and $m$ are the momentum and mass of the electron in some atom, $u(x)$ is the Coulomb potential of interaction of the electron with nucleus, and $\vec{A}$ is the vector potential of the secondly quantized electromagnetic field which is given by the formula

$$
\vec{A}=\sum_{k}\left(\frac{2 \pi c^{2}}{\omega_{k}}\right)^{1 / 2} \vec{e}_{k}\left(b_{k}^{+} e^{-i \vec{k} \vec{x}}+b_{k} e^{i \vec{k} \vec{x}}\right), \quad\left[b_{k}, b_{k^{\prime}}^{+}\right]=\delta_{k k^{\prime}},
$$

where $\vec{e}_{k}$ are polarization vectors and $b_{k}, b_{k}^{+}$are the usual operators of annihilation and creation of the mode with wave vector $\vec{k}$ and the energy $\omega_{k}$. There are several steps of simplification of this general Hamiltonian. First, one neglects the term which is nonlinear in the vector potential, $\frac{e^{2}}{2 m c^{2}} \vec{A}^{2}$. Second, one considers only transitions between higher levels of an atom,

$$
\frac{1}{2 m} \vec{p}^{2}+u(x) \rightarrow \omega_{0} \sigma^{z}+\text { const }
$$

`This paper is a contribution to the Vadim Kuznetsov Memorial Issue "Integrable Systems and Related Topics". The full collection is available at http://www.emis.de/journals/SIGMA/kuznetsov.html 
where $\sigma^{z}$ is the Pauli matrix. Third, one considers only small $\vec{k}, k a \ll 1$, where $a$ is the typical size of an atom, $e^{ \pm i \vec{k} \vec{x}} \sim 1$. Fourth, one uses the dipole and so-called rotating wave approximations [4], which result in

$$
-\frac{e}{m c} \vec{p} \vec{A} \rightarrow \sum_{k} g_{k} \sigma^{x}\left(b_{k}^{+}+b_{k}\right) \quad \rightarrow \quad \sum_{k} g_{k}\left(b_{k}^{+} \sigma^{-}+b_{k} \sigma^{+}\right),
$$

where $\sigma^{ \pm}$are the usual Pauli matrices. And finally, one considers only the case of resonance: $\sum_{k} \rightarrow k_{\text {fixed. }}$. By all these steps one can write down very simple Jaynes-Cummings Hamiltonian [2] which is linear in the operators of creation and annihilation of one resonant mode,

$$
H_{J C}=\omega_{0} b^{+} b+\frac{1}{2} \omega_{0} \sigma^{z}+g\left(\sigma^{+} b+\sigma^{-} b^{+}\right) .
$$

The solution of eigenequation for the operator (1) is almost trivial, but it allows one to describetime evolution of the corresponding wave function in great detail, with transparent applications to physics of one-mode laser. This extremely simple model admits almost evident generalisations in two directions.

\section{The simplest generalisations of the JC model: the $n$-level atom and Dicke model}

The first of these generalisations is the model of one $n$-level atom with $(n-1)$ modes of resonant radiation,

$$
H_{J C}^{(n)}=\sum_{\alpha=1}^{n-1} \omega_{\alpha} \hat{N}_{\alpha}+\Delta \hat{R}_{n n}+\sum_{\alpha=1}^{n-1} g_{\alpha}\left(b_{\alpha} \hat{R}_{n \alpha}+b_{\alpha}^{+} \hat{R}_{\alpha n}\right),
$$

where

$$
\hat{N}_{\alpha}=b_{\alpha}^{+} b_{\alpha}-\hat{R}_{\alpha \alpha}, \quad\left(\hat{R}_{\alpha \beta}\right)_{\gamma \delta}=\delta_{\alpha \gamma} \delta_{\beta \delta},
$$

the sets $\left\{\omega_{\alpha}\right\},\left\{g_{\alpha}\right\}$ are energies of the modes and their coupling constants, and $\Delta$ is so-called detuning parameter $[5,6]$. The extended physical motivation for this kind of the generalization of the Jaynes-Cummings model can be found in [6]. There are evident commutation relations

$$
\left[\hat{N}_{\alpha}, \hat{N}_{\beta}\right]=0, \quad\left[H_{J C}^{(n)}, \hat{N}_{\alpha}\right]=0,
$$

which make the problem of diagonalisation of (2) at $n \geq 3$ quite easy. The second way of generalisation of the Jaynes-Cummings model consists in considering an arbitrary number $N$ of two-level sources of radiation,

$$
H_{D}=\omega b^{+} b+\sum_{j=1}^{N}\left[\omega_{j} \sigma_{z}^{(j)}+\lambda\left(\sigma_{+}^{(j)} b+\sigma_{-}^{(j)} b^{+}\right)\right] .
$$

The solution of this so-called Dicke model which has been proposed many years ago [1] is far from being trivial. It turned out that the problem with the Hamiltonian (3) has some hidden dynamical symmetry which allows one to construct some nontrivial operators commuting with (3).

What happens if one will try to use both the ways of generalisation of the Jaynes-Cummings model, i.e. consider the Hamiltonian of the problem of an arbitrary number $N$ of multilevel sources interacting with an arbitrary number $n-1$ of resonant modes of radiation,

$$
H^{(N, n)}=\sum_{\alpha=1}^{n-1} \omega_{\alpha} \hat{N}_{\alpha}+\sum_{k=1}^{N} \Delta_{k} \hat{R}_{n n}^{(k)}+\sum_{\alpha=1}^{n-1} \sum_{k=1}^{N} g_{\alpha}^{(k)}\left[b_{\alpha} \hat{R}_{n \alpha}^{(k)}+b_{\alpha}^{+} \hat{R}_{\alpha n}^{(k)}\right],
$$


where $N \geq 2, n \geq 3$ (the detuning parameters $\{\Delta\}$ and coupling constants $\left\{g_{\alpha}^{(k)}\right\}$ are assumed to be arbitrary)? The answer is not clear till now. In the following two sections we will argue that there is some simplification of the spectral problem defined by (4) in the case of equality of all the modules of the coupling constants $\{g\}$ : these constants should not depend on $k$ and their dependence on $\alpha$ is given by the formula $g_{\alpha}^{(k)} \propto \varepsilon_{\alpha}, \varepsilon_{\alpha}= \pm 1$. Namely, we will construct in this case explicitly some set of $N-1$ nontrivial operators commuting with (4); their existence allows one to call the model (4) quantum integrable under the above choice of the coupling constants.

\section{The models of Gaudin type}

In this section, let us consider some more general operators introduced first by Gaudin [7],

$$
\mathcal{H}_{j}^{(N)}=\sum_{\alpha, \beta=1}^{n} \sum_{s \neq j}^{N+1} f_{j s}^{\alpha \beta} \hat{T}_{\alpha \beta}^{j} \hat{T}_{\beta \alpha}^{s}, \quad j=1, \ldots, N+1,
$$

where $\left\{\hat{T}_{\alpha \beta}^{j}\right\}$ are operators of absolutely arbitrary representations of $S L(n)$, obeying the commutation relations

$$
\left[\hat{T}_{\alpha \beta}^{j}, \hat{T}_{\lambda \mu}^{s}\right]=\delta_{j s}\left(\hat{T}_{\alpha \mu}^{j} \delta_{\beta \lambda}-\hat{T}_{\lambda \beta}^{j} \delta_{\alpha \mu}\right),
$$

and $\left\{f_{j s}^{\alpha \beta}\right\}$ are arbitrary sets of numbers, obeying the evident restriction $f_{j s}^{\alpha \beta}=f_{j s}^{\beta \alpha}$. It is easy to see that operators

$$
\hat{N}_{\alpha}=\sum_{j=1}^{N+1} \hat{T}_{\alpha \alpha}^{j}
$$

trivially commute with (5),

$$
\left[\mathcal{H}_{j}^{(N)}, \hat{N}_{\alpha}\right]=0 .
$$

Now let us pose the problem: how to find such the sets of numbers $\{f\}$ that

$$
\left[\mathcal{H}_{j}^{(N)}, \mathcal{H}_{k}^{(N)}\right]=0
$$

for all indices $\{j, k\}$. It is easy to see that in this case the "Hamiltonian"

$$
H_{n}^{(N)}=\sum_{j=1}^{N+1} \eta_{j} \mathcal{H}_{j}^{(N)}+\sum_{\alpha=1}^{n} \omega_{\alpha} \hat{N}_{\alpha}
$$

where $\eta_{j}$ and $\omega_{\text {alpha }}$ are arbitrary constants, commutes with all $\mathcal{H}_{j}^{(N)}$,

$$
\left[H_{n}^{(N)}, \mathcal{H}_{j}^{(N)}\right]=0 .
$$

In the simplest nontrivial case of $n=2$, one can always choose $\{f\}$ such that $f_{j k}^{\alpha \beta}=-f_{k j}^{\beta \alpha}$, and the equations (7) can be written in the form

$$
\begin{aligned}
{\left[\mathcal{H}_{j}^{(N)}, \mathcal{H}_{k}^{(N)}\right]=} & -\sum_{s \neq j, k}^{N+1} \sum_{\alpha, \beta, \lambda=1}^{n}\left[f_{j s}^{\alpha \beta} f_{s k}^{\alpha \lambda}+f_{k j}^{\alpha \lambda} f_{j s}^{\beta \lambda}+f_{j k}^{\alpha \beta} f_{k s}^{\beta \lambda}\right] \\
& \times\left(\hat{T}_{\alpha \beta}^{j} \hat{T}_{\lambda \alpha}^{k} \hat{T}_{\beta \lambda}^{s}-\hat{T}_{\beta \alpha}^{j} \hat{T}_{\alpha \lambda}^{k} \hat{T}_{\lambda \beta}^{s}\right)=0
\end{aligned}
$$


which immediately gives a overdetermined set of bilinear equations for $\{f\}$,

$$
f_{j s}^{\alpha \beta} f_{s k}^{\alpha \lambda}+f_{k j}^{\alpha \lambda} f_{j s}^{\beta \lambda}+f_{j k}^{\alpha \beta} f_{k s}^{\beta \lambda}=0 .
$$

It has been shown by Gaudin $[7,8]$ that for $n=2$ there is some so-called anisotropic solution to $(10)$,

$$
f_{j s}^{\alpha \alpha}=\cot \left(u_{j}-u_{s}\right), \quad f_{j s}^{\alpha \beta}=\frac{1}{\sin \left(u_{j}-u_{s}\right)},
$$

where $\left\{u_{j}\right\}$ are arbitrary numbers. Now, following Gaudin, let us choose them as

$$
u_{k}=-\varepsilon \lambda_{k}+\frac{\pi}{2}+u_{N+1}, \quad k=1, \ldots, N .
$$

As $\varepsilon \rightarrow 0$, then $f_{j, N+1}^{\alpha \alpha} \sim \varepsilon \lambda_{j}, f_{j, N+1}^{\alpha \beta} \sim 1$. Let us choose $\left\{\hat{T}_{\alpha \beta}^{N+1}\right\}$ as Jordan-Schwinger realization of $S L(2), \hat{T}_{\alpha \beta}^{N+1}=-b_{\alpha} b_{\beta}^{+}$and consider the limit $N_{2} \gg 1$, where $N_{2}$ is an eigenvalue of the operator $\hat{N}_{2}$. It is easy to see that in this limit $b$-operators can be treated as $c$-numbers; $b_{2}, b_{2}^{+} \sim{\sqrt{N_{2}}}_{2}$; let us also take $\varepsilon \sim N_{2}^{\frac{-1}{2}}$ and send $N_{2} \rightarrow \infty$. In this limiting process, one easily finds

$$
\lim _{N_{2} \rightarrow \infty} H_{2}^{(N)}=\omega_{1} b_{1}^{+} b_{1}+\sum_{j=1}^{N}\left[\lambda_{j}\left(\hat{T}_{11}^{(j)}-\hat{T}_{22}^{(j)}\right)+\left(\hat{T}_{12}^{(j)} b_{1}+\hat{T}_{21}^{(j)} b_{1}^{+}\right)\right] .
$$

And finally, if we will choose $\left\{\hat{T}_{\alpha \beta}^{(j)}\right\}$ as $\sigma$-representation of $S L(2)$, we will obtain just the Dicke Hamiltonian!

This derivation of the Dicke model proposed first by Gaudin [7] is not original of course. There are some recent papers in which the connection between it and the Gaudin models was exploited for construction the algebraic Bethe ansatz method for eigenvectors of the Dicke Hamiltonian and some generalisations which might be considered as the interaction term without the rotating wave approximation $[9,10,11]$.

The above limiting procedure, however, does not work in the general case $n \geq 3$. One also has in this case the overdetermined system of bilinear equations

$$
f_{j s}^{\alpha \beta} f_{s k}^{\alpha \lambda}+f_{k j}^{\alpha \lambda} f_{j s}^{\beta \lambda}+f_{j k}^{\alpha \beta} f_{k s}^{\beta \lambda}=0,
$$

but, if $n \geq 3$, there is a possibility of the choice of indices $\alpha \neq \beta \neq \lambda$ which excludes all "anisotropic" solutions of the type (11). There are only "isotropic" solutions of the type $f_{j s}^{\alpha \alpha}=$ $f_{j s}^{\alpha \beta}=\frac{1}{\nu_{j}-\nu_{s}}$, and hence there are no parameters for $N_{n} \rightarrow \infty$. Hence it seems that the Gaudin approach does not lead to any integrable system of the type (4).

Let us prove absence of "anisotropic" solutions to (10) as $N=2, n \geq 3$. In this simplest nontrivial case the set $\{f\}$ can be parametrized as

$$
f_{j s}^{\alpha \beta}=\sum_{p=1}^{3} \varepsilon_{j s p} A_{p}^{\alpha \beta}, \quad f_{s k}^{\alpha \lambda}=\sum_{p=1}^{3} \varepsilon_{s k p} A_{p}^{\alpha \lambda}, \quad f_{j s}^{\beta \lambda}=\sum_{p=1}^{3} \varepsilon_{j s p} A_{p}^{\beta \lambda} .
$$

As $j \neq k \neq s,(10)$ reads:

$$
A_{k}^{\alpha \beta} A_{j}^{\alpha \lambda}+A_{s}^{\alpha \lambda} A_{k}^{\beta \lambda}+A_{s}^{\alpha \beta} A_{j}^{\beta \lambda}=0,
$$

with general solution

$$
A_{1}^{\alpha \lambda}=\frac{A}{\sin \nu_{1}}, \quad A_{2}^{\alpha \lambda}=\frac{A}{\sin \nu_{2}}, \quad A_{3}^{\alpha \lambda}=-\frac{A}{\sin \left(\nu_{1}+\nu_{2}\right)},
$$




$$
A_{1}^{\alpha \alpha}=A \cot \nu_{1}, \quad A_{2}^{\alpha \alpha}=A \cot \nu_{2}, \quad A_{3}^{\alpha \alpha}=-A \cot \left(\nu_{1}+\nu_{2}\right),
$$

where $A, \nu_{1}, \nu_{2}$ are some parameters. Now, if one takes $\alpha \neq \beta \neq \lambda$, one gets from (10)

$$
-\frac{A^{2}}{2 \cos \frac{n u_{1}}{2} \cos \frac{\nu_{2}}{2} \cos \frac{\nu_{1}+\nu_{2}}{2}}=0 \quad \rightarrow \quad A=0 .
$$

Surprisingly enough, one can construct integrable models of Gaudin type at $n \geq 3$ by using only "isotropic" solution of (10). It will be done in the next section.

\section{New integrable model with $N$ sources and $n-1$ modes}

The receipt of constructing the integrable model of the type (4) is quite unusual. Let us choose the above $\mathcal{H}$-operators as obeying the equality

$$
\left[\mathcal{H}_{j}^{(N)}, \mathcal{H}_{k}^{(N)}\right]=\hat{S}_{j k}\left(\left\{\hat{T}_{\alpha \beta}^{s}\right\},\{x\}\right),
$$

where $x$ is some parameter and $\hat{S}_{j k}$ have the following property: as $x \rightarrow \infty$, there are only zero eigenvalues of them in a subspace in which $\left\{\hat{T}_{\alpha \beta}^{s}\right\}$ act nontrivially. Then we can introduce new operators $\left(\lim _{x \rightarrow \infty} \mathcal{H}_{j}^{(N)}\right)$ in this subspace which will commute, and $H_{n}^{N+1}=-\sum_{j=1}^{N} \mathcal{H}_{j}^{(N)}$ is some nontrivial Hamiltonian. By direct calculation of the commutator of the operators (5), one finds

$$
\begin{aligned}
& \hat{S}_{j k}\left(\left\{\hat{T}_{\alpha \beta}^{s}\right\},\{x\}\right\}=-\sum_{s \neq j, k}^{N} \sum_{\alpha, \beta, \lambda=1}^{n}\left(f_{j s}^{\alpha \beta} f_{s k}^{\alpha \lambda}+f_{k j}^{\alpha \lambda} f_{j s}^{\beta \lambda}+f_{j k}^{\alpha \beta} f_{k s}^{\beta \lambda}\right) \\
& \quad \times\left(\hat{T}_{\alpha \beta}^{j} \hat{T}_{\lambda \alpha}^{k} \hat{T}_{\beta \lambda}^{s}-\hat{T}_{\beta \lambda}^{j} \hat{T}_{\alpha \lambda}^{k} \hat{T}_{\lambda \beta}^{s}\right)-\sum_{\alpha, \beta, \lambda=1}^{n}\left(f_{j, N+1}^{\alpha \beta} f_{N+1, k}^{\alpha \lambda}+f_{k j}^{\alpha \lambda} f_{j, N+1}^{\beta \lambda}+f_{j k}^{\alpha \beta} f_{k, N+1}^{\beta \lambda}\right) \\
& \quad \times\left(\hat{T}_{\alpha \beta}^{j} \hat{T}_{\lambda \alpha}^{k} \hat{T}_{\beta \lambda}^{N+1}-\hat{T}_{\beta \lambda}^{j} \hat{T}_{\alpha \lambda}^{k} \hat{T}_{\lambda \beta}^{N+1}\right) .
\end{aligned}
$$

Let us choose $f_{j k}^{\alpha \beta}=A\left(\nu_{j}-\nu_{k}\right)^{-1}, 1 \leq j, k \leq N$, i.e. use the isotropic Gaudin solution at $j, s, k \leq N$. Then the double sum vanishes and we are left with

$$
\begin{aligned}
\hat{S}_{j k}\left(\left\{\hat{T}_{\alpha \beta}^{s}\right\},\{x\}\right)= & -\sum_{\alpha, \beta, \lambda=1}^{n}\left[f_{j, N+1}^{\alpha \beta} f_{N+1, k}^{\alpha \lambda}+\frac{A}{\nu_{j}-\nu_{k}}\left(f_{k, N+1}^{\beta \lambda}-f_{j, N+1}^{\beta \lambda}\right)\right] \\
& \times\left(\hat{T}_{\alpha \beta}^{j} \hat{T}_{\lambda \alpha}^{k} \hat{T}_{\beta \lambda}^{N+1}-\hat{T}_{\beta \lambda}^{j} \hat{T}_{\alpha \lambda}^{k} \hat{T}_{\lambda \beta}^{N+1}\right) .
\end{aligned}
$$

There are no other restrictions to the numbers $\left\{f_{j, N+1}^{\alpha \beta}\right\}$ except symmetry. Hence we have $\frac{N n(n+1)}{2}$ free parameters at given $\left\{A,\left\{\nu_{j}\right\}\right\}$ in the relation (16). Of course, there is trivial case if all $f_{j, N+1}^{\alpha \beta}=0$. Fortunately, one can construct the nontrivial ansatz:

$$
f_{j, N+1}^{\alpha \beta}=\left(\delta_{\beta n} f_{j, N+1}^{\alpha n}+\delta_{\alpha n} f_{j, N+1}^{\beta n}\right)\left(1-\delta_{\alpha n} \delta_{\beta n}\right) f_{j, N+1}^{n n},
$$

i.e. $f_{j, N+1}^{\alpha \beta}=0$ unless $\alpha \neq n$ or $\beta \neq n$.

The commutator $\left[\mathcal{H}_{j}^{N}, \mathcal{H}_{k}^{N}\right](16)$ is still too complicated. Let us make the next assumption:

$$
f_{j, N+1}^{\lambda n}=\varepsilon_{\lambda} \sqrt{A F}, \quad f_{j, N+1}^{n n}=-F \nu_{j}, \quad\left|\varepsilon_{\lambda}\right|=1,
$$


where $\left\{\nu_{j}\right\}$ are arbitrary numbers. Then (16) can be recast in the form

$$
\begin{aligned}
{\left[\mathcal{H}_{j}^{(N)}, \mathcal{H}_{k}^{(N)}\right]=} & \sum_{\beta, \lambda=1}^{n-1} A F \varepsilon_{\beta} \varepsilon_{\lambda}\left(\hat{T}_{n \beta}^{j} \hat{T}_{\lambda n}^{k} \hat{T}_{\beta \lambda}^{N+1}-\hat{T}_{\beta n}^{j} \hat{T}_{n \lambda}^{k} \hat{T}_{\lambda \beta}^{N+1}\right) \\
& -\sum_{\lambda=1}^{n-1} \sqrt{A F}\left\{F \nu_{j} \varepsilon_{\lambda}\left(\hat{T}_{n n}^{j} \hat{T}_{\lambda n}^{k} \hat{T}_{n \lambda}^{N+1}-\hat{T}_{n n}^{j} \hat{T}_{n \lambda}^{k} \hat{T}_{\lambda n}^{N+1}\right)\right. \\
& \left.-F \nu_{k} \varepsilon_{\lambda}\left(\hat{T}_{n \lambda}^{j} \hat{T}_{n n}^{k} \hat{T}_{\lambda n}^{N+1}-\hat{T}_{\lambda n}^{j} \hat{T}_{n n}^{k} \hat{T}_{n \lambda}^{N+1}\right)\right\} .
\end{aligned}
$$

Let now choose

$$
\hat{T}_{\beta \lambda}^{N+1}=-b_{\beta} b_{\lambda}^{+}
$$

as Jordan-Schwinger representation of $S L(n))$. Hence the basis of the whole Hilbert space of the problem consists of the direct products of the basis vectors of the spaces in which $\hat{T}_{\beta \lambda}^{j}, 1 \leq j \leq N$ act, and various basic vectors of above Jordan-Schwinger representation, $\left\{\prod_{\beta,\{l\}}\left(b_{\beta}^{+}\right)^{l_{\beta}}|0\rangle\right\}$, $\{l\} \in \mathbb{N}$. Consider the action of the right-hand side of (17) on the subspace spanned by the vectors $\varphi_{n n}^{(L)}$ with $l_{n}=L, L \gg 1$ such that

$$
\hat{N}_{n} \varphi_{n n}^{(L)} \sim L^{2} \varphi_{n n}^{(L)}, \quad \hat{N}_{n} \varphi_{n n}^{(L)}=-\left(\hat{T}_{n n}^{N+1}+\sum_{j=1}^{N} \hat{T}_{n n}^{j}\right)=b_{n} b_{n}^{+}-\sum_{j=1}^{N} \hat{T}_{n n}^{j} .
$$

Then

$$
\hat{T}_{n n}^{N+1} \sim-L^{2}, \quad \hat{T}_{n \alpha}^{N+1} \sim-L b_{\alpha}^{+}, \quad \hat{T}_{\alpha n}^{N+1} \sim-L b_{\alpha}, \quad 1 \leq \alpha \leq n-1 .
$$

If

$$
F=\frac{A \Delta}{L^{2}}, \quad f_{k, N+1}^{n n}=-\frac{A \Delta \nu_{k}}{L^{2}}, \quad f_{k, N+1}^{\lambda n}=\frac{\varepsilon_{\lambda} A \sqrt{\Delta}}{L},
$$

then

$$
\lim _{L \rightarrow \infty}\left[\mathcal{H}_{j}^{(N)}, \mathcal{H}_{k}^{(N)}\right] \varphi_{n n}^{(L)}=0
$$

and

$$
\tilde{\mathcal{H}}_{j}^{(N)}=\lim _{L \rightarrow \infty} \mathcal{H}_{j}^{(N)}
$$

commute on this subspace! The explicit form of $\mathcal{H}$-operators reads

$$
\begin{aligned}
& \tilde{\mathcal{H}}_{j}^{(N)}=A \sum_{s \neq j}^{N} \sum_{\alpha, \beta=1}^{n} \hat{T}_{\alpha \beta}^{j} \hat{T}_{\beta \alpha}^{s}\left(\nu_{j}-\nu_{s}\right)^{-1}+\hat{w}_{j}, \\
& \hat{w}_{j}=A\left[\sum_{\lambda=1}^{n-1} \varepsilon_{\lambda} \sqrt{\Delta}\left(b_{\lambda} \hat{T}_{n \lambda}^{j}+b_{\lambda}^{+} \hat{T}_{\lambda n}^{j}\right)+\Delta \nu_{j} \hat{T}_{n n}^{j}\right], \\
& H_{N+1}^{(N)}=-\sum_{j=1}^{(N)} \tilde{\mathcal{H}}_{j}^{(N)}=-\sum_{j=1} \hat{w}_{j}=-A \sum_{k=1}^{N}\left[\sum_{\lambda=1}^{n-1} \varepsilon_{\lambda} \sqrt{\Delta}\left(b_{\lambda} \hat{T}_{n \lambda}^{k}+b_{\lambda}^{+} \hat{T}_{\lambda n}^{k}\right)+\Delta \nu_{k} \hat{T}_{n n}^{k}\right] .
\end{aligned}
$$

They commute with $\left\{\tilde{\mathcal{H}}_{j}^{(N)}\right\}$ and $\hat{N}_{\alpha}=-\sum_{j=1}^{N} \hat{T}_{\alpha \alpha}^{j}+b_{\alpha}^{+} b_{\alpha}$. 
And finally, let us choose as $\hat{T}_{\alpha \beta}^{j}$ the matrix representation of $S L(n)$,

$$
\hat{T}_{\alpha \beta}^{j} \rightarrow \hat{R}_{\alpha \beta}^{j},
$$

and add linear combination $\sum_{\alpha=1}^{n-1} \omega_{\alpha} \hat{N}_{\alpha}$ to $H_{N+1}^{(N)}$.

It is easy to see that we obtain just the operator of generalized Dicke model (4) for $n \geq 3$,

$$
H^{(N, n)}=\sum_{\alpha=1}^{n-1} \omega_{\alpha} \hat{N}_{\alpha}+\sum_{k=1}^{N} \Delta_{k} \hat{R}_{n n}^{(k)}+\sum_{\alpha=1}^{n-1} \sum_{k=1}^{N} g_{\alpha}^{(k)}\left[b_{\alpha} \hat{R}_{n \alpha}^{(k)}+b_{\alpha}^{+} \hat{R}_{\alpha n}^{(k)}\right]
$$

with coupling constants

$$
g_{\alpha}^{(k)}=-A \varepsilon_{\alpha} \sqrt{\Delta}, \quad \varepsilon_{\alpha}= \pm 1 .
$$

Hence, under the above choice of the coupling constants, the most general model (4) becomes quantum integrable and the spectral problem might be simplified. However, we did not find the way for doing it except the simplest nontrivial case of $N=2$ which is described in the next section.

\section{Explicit solution for $N=2$}

In this case the Hamiltonian (4) under the conditions (20) can be written as

$$
\begin{aligned}
& H_{n}^{(2)}=\sum_{\alpha=1}^{n-1} \omega_{\alpha} \hat{N}_{\alpha}+\Delta_{1} \hat{R}_{n n}^{1}+\Delta_{2} \hat{R}_{n n}^{2}+\sum_{\alpha=1}^{n-1} \sum_{k=1}^{2}\left[b_{\alpha} \hat{R}_{n \alpha}^{k}+b_{\alpha}^{+} \hat{R}_{\alpha n}^{k}\right], \\
& \hat{N}^{\alpha}=b_{\alpha}^{+} b_{\alpha}-\sum_{j=1}^{2} \hat{R}_{\alpha \alpha}^{j} .
\end{aligned}
$$

Let us rewrite (21) as

$$
\begin{aligned}
& H_{n}^{(2)}=\sum_{\alpha=1}^{n-1} \omega_{\alpha} \hat{N}_{\alpha}+\hat{k}_{1}+\hat{k}_{2} \\
& \hat{k}_{1,2}= \pm\left(\Delta_{1}-\Delta_{2}\right)^{-1} \hat{P}_{1,2}+\sum_{\alpha=1}^{n-1}\left[b_{\alpha} \hat{R}_{n \alpha}^{1,2}+b_{\alpha}^{+} \hat{R}_{\alpha n}^{1,2}\right]+\Delta_{1,2} \hat{R}_{n n}^{1,2}, \quad \hat{P}_{1,2}=\sum_{\alpha, \beta=1}^{n} \hat{R}_{\alpha \beta}^{1} \hat{R}_{\beta \alpha}^{2} .
\end{aligned}
$$

Eigenfunctions of (22) are of course common eigenfunctions of $(n+1)$ operators $\left\{N_{\alpha}\right\}, \hat{k}_{1}, \hat{k}_{2}$,

$$
\psi=\left[\sum_{\lambda, \mu=1}^{n-1} A_{\lambda \mu} \varphi_{\lambda}^{1} \varphi_{\mu}^{2} b_{\lambda}^{+} b_{\mu}^{+}+\sum_{\lambda=1}^{n-1}\left(B_{\lambda}^{(1)} \varphi_{\lambda}^{1} \varphi_{n}^{2}+B_{\lambda}^{(2)} \varphi_{\lambda}^{2} \varphi_{n}^{1}\right) b_{\lambda}^{+}+C \varphi_{n}^{1} \varphi_{n}^{2}\right] Z_{n}|0\rangle,
$$

where $\varphi_{\lambda}^{1}, \varphi_{\mu}^{2}$ are eigenvectors of $\left\{\hat{R}_{\lambda \lambda}^{1}\right\},\left\{\hat{R}_{\mu \mu}^{2}\right\}$ and $Z_{n}=\prod_{\alpha=1}^{n-1}\left(b_{\alpha}^{+}\right)^{N_{\alpha}}$.

One gets from the relations $\hat{k}_{1} \psi=k_{1} \psi, \hat{k}_{2} \psi=k_{2} \psi$ the following set of algebraic equations for the coefficients $\{A, B, C\}$,

$$
\begin{aligned}
& A_{\mu \lambda}\left(\Delta_{1}-\Delta_{2}\right)^{-1}+B_{\mu}^{(2)}=k_{1} A_{\lambda \mu}, \\
& B_{\lambda}^{(2)}\left(\Delta_{1}-\Delta_{2}\right)^{-1}+C=k_{1} B_{\lambda}^{(1)},
\end{aligned}
$$




$$
\begin{aligned}
& B_{\lambda}^{(1)}\left(\Delta_{1}-\Delta_{2}\right)^{-1}+\Delta_{1} B_{\lambda}^{(2)}+\sum_{\mu} A_{\mu \lambda}\left(N_{\mu}+1+\delta_{\lambda \mu}\right)=k_{1} B_{\lambda}^{(2)}, \\
& C\left(\Delta_{1}-\Delta_{2}\right)^{-1}+C \Delta_{1}+\sum_{\mu} B_{\mu}^{(1)}\left(N_{\mu}+1\right)=k_{1} C, \\
& -A_{\mu \lambda}\left(\Delta_{1}-\Delta_{2}\right)^{-1}+B_{\lambda}^{(1)}=k_{2} A_{\lambda \mu}, \\
& -B_{\lambda}^{(1)}\left(\Delta_{1}-\Delta_{2}\right)^{-1}+C=k_{2} B_{\lambda}^{(2)}, \\
& -B_{\lambda}^{(2)}\left(\Delta_{1}-\Delta_{2}\right)^{-1}+\Delta_{2} B_{\lambda}^{(1)}+\sum_{\mu} A_{\lambda \mu}\left(N_{\mu}+1+\delta_{\lambda \mu}\right)=k_{2} B_{\lambda}^{(1)}, \\
& -C\left(\Delta_{1}-\Delta_{2}\right)^{-1}+\Delta_{2} C+\sum_{\mu} B_{\mu}^{(2)}\left(N_{\mu}+1\right)=k_{2} C .
\end{aligned}
$$

They can be easily reduced to two equations for $k_{1}, k_{2}$ :

$$
\begin{aligned}
& \left(k_{1}+k_{2}-\Delta_{1}\right)\left(\Delta_{1}-\Delta_{2}\right)^{-1}+\left(\Delta_{1}-\Delta_{2}\right)^{-2}-k_{1}\left(k_{1}-\Delta_{1}\right)+1+\sum_{\mu=1}^{n-1}\left(N_{\mu}+1\right)=0, \\
& \left(\Delta_{2}-k_{1}-k_{2}\right)\left(\Delta_{1}-\Delta_{2}\right)^{-1}+\left(\Delta_{1}-\Delta_{2}\right)^{-2}-k_{2}\left(k_{2}-\Delta_{2}\right)+1+\sum_{\mu=1}^{n-1}\left(N_{\mu}+1\right)=0,
\end{aligned}
$$

and the eigenvalues of the Hamiltonian (21) are given by

$$
h^{2, n}=\sum_{\alpha=1}^{n-1} \omega_{\alpha} N_{\alpha}+k_{1}+k_{2} .
$$

\section{Conclusions}

In this paper, we found the most general quantum integrable model of interaction of $(n-1)$ modes of radiation with $N$ dipole sources which comprises all known models of that type: JaynesCummings model $(N=1, n=2)$, its generalisation for arbitrary number of modes $(N=1)$, Dicke model (arbitrary $N, n=2)$. The commutative ring of operators which includes $H_{n}^{(N)}$ is found in explicit form. We did not use any "anisotropic" form of the Gaudin solution; the Hamiltonian of the model was obtained via some limiting procedure. We almost immediately got solution for $N=2$, arbitrary $n$ with the use of additional integrals of motion, but the most interesting case of solution for arbitrary $N$ and $\mathrm{n}$ is unreachable at the present stage of investigation. We hope to come back to this problem in the future.

This paper has been presented for memorial volume of Vadim Kuznetsov. We both knew him personally - he was our guest in Dubna almost 17 years ago when he was a PhD student of Professor I.V. Komarov in Leningrad. We remember him as bright young man, very active in the field of quantum and classical integrable systems. Later he solved very complicated problem of integration of equations of motion for classical Toda chains with non-exponential ends [12] proposed by one of us (V.I.).

[1] Dicke R., Coherence in spontaneous radiation processes, Phys. Rev., 1953, V.93, 99-108.

[2] Cummings F.W., Stimulated emission of radiation in a single mode, Phys. Rev. A, 1965, V.140, $1051-1062$.

[3] Heitler W., The quantum theory of radiation, New York, Oxford, 1954.

[4] Allen L., Eberly J., Optical resonance and two-level atoms, New York, Wiley-Interscience Publication, 1975.

[5] Li X., Bei N., A generalized three-level Jaynes-Cummings model, Phys. Lett. A, 1984, V.101, $169-175$. 
[6] Abdel-Hafez A., Obada A., Ahmad M., $N$-level atom and $N-1$ modes: statistical aspects and interaction with squeezed light, Phys. Rev. A, 1987, V.35, 1634-1647.

[7] Gaudin M., Diagonalisation d'une classe d'hamiltoniens de spin, J. de Physique, 1976, V.37, 1087-1098.

[8] Gaudin M., La fonction d'onde de Bethe, Paris, Masson, 1983.

[9] Jurco B., On quantum integrable models related to nonlinear quantum optics. An algebraic Bethe ansatz approach, J. Math. Phys., 1989, V.30, 1739-1744.

[10] Dukelsky J., Dussel G.G., Esebbag C., Pittel S., Exactly solvable models for atom-molecule Hamiltonians, Phys. Rev. Lett., 2004, V.93, 050403, 4 pages, cond-mat/0406190.

[11] Kundu A., Integrable multi-atom matter-radiation models without rotating-wave approximation, Phys. Lett. A, 2006, V.350, 210-213, cond-mat/0411166.

[12] Kuznetsov V.B., Separation of variables for the $\mathcal{D}_{n}$-type periodic Toda lattice, J. Phys. A: Math. Gen., 1997, V.30, 2127-2138, solv-int/9701009. 